\title{
Opportunities for autonomous UAV in harsh environments
}

\author{
R. La Scalea*, M. Rodrigues*, D. P. M. Osorio ${ }^{\dagger}$, C. H. Lima ${ }^{\ddagger}$, R. D. Souza ${ }^{\S}$, H. Alves ${ }^{\ddagger}$, and K. C. Branco* \\ *ICMC, University of São Paulo, São Carlos, Brazil \\ $\dagger^{\dagger}$ Department of Electrical Engineering, Federal University of São Carlos, São Carlos, Brazil \\ $\ddagger 6$ G Flagship, University of Oulu, Finland \\ $\S_{\text {Federal University of Santa Catarina, Florianópolis, Brazil }}$ \\ E-mails: la.scalea@live.com, rodrigues.mariana@gmail.com, dianamoya@ufscar.br, carlos.lima@oulu.fi, richard.demo@ufsc.br, hirley.alves@oulu.fi, kalinka@icmc.usp.br
}

\begin{abstract}
This paper describes the challenges raised from the use of Unmanned Aerial Vehicles (UAVs) on the exploration of inhospitable places that present limitations and risks to humans; for instance, we describe the case of cave exploration. UAVs have emerged as an attractive alternative for performing this task in a safety and reliable manner. However, challenges regarding control management, accurate positioning and navigation, and reliable wireless connectivity need to be addressed in order to achieve effective solutions. Herein, we address those challenges and present possible solutions for enabling the use of UAVs in harsh environments.
\end{abstract}

Index Terms-harsh environment, location and positioning, multipath channels, Unmanned Aerial Vehicles.

\section{INTRODUCTION}

An Unmanned Aerial Vehicle (UAV) is an aircraft that can fly without any human input or supervision. Usually, they are part of an Unmanned Aerial System (UAS) together with a Ground Control Station (GCS) and the communication links [1]. Historically, they were mostly employed in military applications, but have been gaining ground in civilian applications such as precision agriculture, track monitoring, surveillance, logistics, search-rescue missions, among others [2]. UAVs are usually very resource-constrained, greatly impacting aspects such as maximum flight time, communication efficiency and data processing and storage. Energy-efficiency must be taken into account in any UAV-based solution to maximize battery life and flight time.

There is a growing interest on using UAVs as a lowcost technology for providing connectivity in indoors environments, specially for industry applications. UAVs can also be used to reach dangerous places where humans cannot access in safety conditions [3]. However, missions over confined

This work was partially supported by the Coordenação de Aperfeiçoamento de Pessoal de Nível Superior - Brasil (CAPES) - Finance Code 001, the São Paulo Research Foundation (FAPESP) - Proc. No 2017/20990-6, and by Academy of Finland 6Genesis Flagship (Grant no. 318927), Aka Project EEIoT (Grant no. 319008).

Research was also sponsored by the Army Research Office and was accomplished under Grant Number W911NF-18-1-0012. The views and conclusions contained in this document are those of the authors and should not be interpreted as representing the official policies, either expressed or implied, of the Army Research Office or the U.S. Government. The U.S. Government is authorised to reproduce and distribute reprints for Government purposes notwithstanding any copyright notation herein. spaces, where absolute positioning signals and remote control are not available or sufficiently precise represent a very challenging task. Particularly, UAV systems in confined environments would need robust wireless communications, threedimensional trajectory data, precise control, and scheduling (UAV's control commands).

Regardless of their size or autonomy level, the state of UAVs is required to be known by its controller or its automatic pilot. Based on the acquired data, the UAV control system can command the actuators so the desired trajectory is met [4]. UAV state comprise its position, velocity, altitude, airspeed, angleof-attack, sideslip angle, and rotation (pitch, roll, and yaw) rates, which are acquired by an Inertial Measurement Unit (IMU) and Global Positioning System (GPS) receiver. IMU measurements present accumulated error over time, which is compensated by the GPS location information [5].

When considering a harsh and confined environment, the navigation and therefore UAV control can be seriously compromised. High-frequency waves cannot penetrate dense obstacles, and in general the GPS system is not available. Therefore, the UAV must either operate remotely within a Line of Sight (LoS) or employ another navigation strategy for a GPS-denied environment [6]. One of the strategies to compensate the lack of GPS signals are fusing IMU and camera data, resulting in a vision-based navigation strategy. Popular strategies for vision-based navigation are the Simultaneous Localization and Mapping (SLAM), which builds an environment map and estimate the UAV position based on the acquired image, and Visual Odometry (VO), which estimates the vehicle's position and orientation based on the sequence of images acquired [5]. However, some environments may have no light to apply a vision-based approach in UAV localization. The use of a lightning source at all times would demand UAV power and decrease its autonomy. Therefore, other localization techniques are required that do not rely on computer vision.

In UAV applications, there is a predominance of decentralized localization strategies based on distance, such as the Received Signal Strength Indication (RSSI) and Time Difference of Arrival (TDOA). RSSI is a technique in which the distance between nodes is estimated using the signal strength and used to calculate the position [7]. This technique was applied for UAVs in GPS-denied environments in [8]. TDOA 
bases its measurement on the difference in the arrival times of a signal coming from multiple sources. This technique, applied in [9] is cost effective and less affected by radio reflection [7]. Ultra-wideband (UWB) technology has attracted interest as a promising solution for accurate location and tracking due to its ability on resolving multipath and penetrating obstacles [10].

In this paper, we discuss the challenges and potentials technologies to address the use of UAVs in harsh environments by considering the special case of cave exploration. Section II describes our case study. Section III presents the main challenges regarding communication, control, and positioning issues. Finally, Section IV presents some final remarks.

\section{CASE Study: CAVE EXPLORATION}

Caves have been present in mankind History since the prehistoric period, being used for shelter, economic activities, storage and ritual purposes [11]. As an example, in Brazil, there are around 6000 identified caves, many of them without speleological studies. Cave surveying (or cave mapping) is an attempt to accurately record the cave and its characteristics [12]. Those maps detail the cave extension, layout and passages, and are useful not only for cave research, but for other knowledge areas such as archaeology, biology, geology, and others [13]. Traditionally, cave mapping has been done in a rudimentary manner, using tools such as compasses or clinometers and taking notes that were used to draw the cave maps [12]. The improvement of available tools such as 3D sensors allowed speleologists to provide better mapping and cave information [14]-[16]. All these strategies, however, still depend on a person entering the cave, which can be hazardous for the speleologist depending on the environment or even impossible if the area to be mapped is unreachable by humans.

In [16], a mobile cave mapping with LiDAR is presented, and in that work is suggested the use of an Unmanned Aerial Vehicle (UAV) integrated to a LiDAR sensor for cave mapping. Digital photogrammetry can also be deployed in UAVs to help speleologists, as demonstrated in [17]. Even though UAVs can bring many benefits to cave surveying due to its access to dangerous and inaccessible sites, there are some factors that hinder the use of those vehicles in these environments. Indeed, non-maintained or natural subterranean environments can be considered one of the most challenging for robotic operations [6], since they can present limited access, have water, gases and wildlife inside them, have no illumination and greatly affected communication. Therefore, robots or Unmanned Vehicles employed in such environments must present higher reliability and robustness.

In general, inhospitable scenarios as those of caves present different rock formations and environments and passages of variable dimensions. In its interior can occur the total absence of light, irregular ground and different kinds and sizes of obstacles such as speleothems, besides the possibility of containing bodies, water and cave fauna, especially bats. Those characteristics represent a very difficult challenge for UAV navigation and manoeuvres. Also, the irregular environment influences the communication signals through reflection, diffraction and scattering, making UAV operation very challenging in these environments. Fig. II shows an example of a cave.

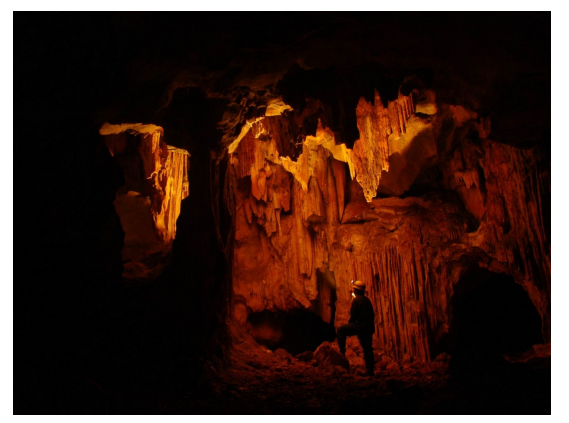

Fig. 1. Interior of the Pinheiro Seco cave, located in the municipality of Castro, in the state of Paraná, Brazil.

\section{Challenges and Potential Directions}

In this section, we describe the main challenges regarding the control of UAVs inside the described environments, the communications and positioning issues as well as the corresponding possible solutions.

\section{A. Challenges in Control}

1) Communication Maintenance: Regardless of whether the drone is autonomous or remotely controlled, the flight control will depend heavily on the communication link maintenance to keep the aircraft stable during flight [18]. Communication disruption in remotely operated UAVs can cause the operator to lose control of the aircraft, which can become unstable and crash. In autonomous UAVs, the autopilot is a key component of the aircraft control system, receiving GPS positioning data and controlling the UAV to follow reference paths, avoid obstacles or navigate through waypoints. In GPSdenied environments (cave scenarios) or if GPS signals outages happens, the navigation system can deteriorate rapidly due to the large sensor errors presented by the low cost microelectromechanical sensors used in most off-the-shelf autopilot systems [19].

2) Delay: In embedded systems, the network represents the interconnections among components (sensors, actuators, controller and ground station) [20]-[22]. Delays on the network can greatly prejudice robust networked control systems, which need reliable real-time information to provide fast, accurate responses. An example can be seen in [23], where network issues such as transmission delay and packet loss can result in a significant threat to the train stability and operation safety. Likewise, delayed UAV information fed to the control system can cause instability and the inability of returning to the initial navigation point or avoiding obstacles inside the environment. Therefore, reducing the delay is a big challenge when working with critical embedded realtime systems. 
3) Noise, Bit Errors, and the Risk of Instability: In control systems with quantized feedback control values being transmitted from a controller to the sensor/actuators over a noisy feedback channel, randomly occurring bit errors will degrade the performance of the system. This degradation will depend on a number of factors, including the channel noise and whether the control levels have been coded to deal with more than a single bit error. With a noisy channel and frequent bit errors, the performance in the close loop system can evolve to the point of instability [24], [25]. When using UAVs inside a cave, a control system instability could represent the lost of the UAV or a possible damage to the cave So it is important to reduce the channel noise and prevent a system instability in order to reap the best benefits from UAV usage.

\section{B. Challenges in Communications}

1) Channel Characterization: Appropriate characterization of channel propagation effects is of paramount importance for the design and analysis of UAV assisted wireless communication networks. There has been a great interest in the research community in measuring and modelling air-to-ground and air-to-air wireless channels - the survey in [26] summarizes the main findings in this area. For instance, fading, path loss, shadowing and Doppler effect have been measured or modelled considering different types of UAVs, carrier frequency and communication technology [27]-[34]. Common to these works is the conclusion that line-of-sight (LOS) plays a great role and that the UAV altitude has a direct relation to the amount of LOS, especially in air-to-ground channels. Moreover, these works focus basically on aerial or over the ground communications, not in underground channels. Although not including UAVs, there is a rich literature devoted to the wireless characterization of tunnels and mines [35]-[38], but the same cannot be said for the case of natural caves. A recent exception is the experimental work reported in [39], which characterizes the wireless channel inside a touristic cave in Malaysia. Although a natural cave differs in many ways from an underground mine, such as depth, width of the paths, type of rocks, among other points, the authors in [39] also report the severe impact of non-line-of-sight (NLOS) conditions induced by the irregular trajectory inside the cave, with several corners. Despite the differences, localization and tracking schemes designed and deployed to work in underground mines (e.g. [40]), should be adaptable to the particular characteristics of wireless propagation inside caves.

2) Diversity and reliability enhancements: In harsh environments, the radio signal can be dramatically degradedby diffraction, reflection and scattering due to obstacles, resulting in several replicas with different amplitudes, delay and Doppler components. This leads to destructive and constructive interference of the different signal versions arriving at the receiver, which make its detection extremely difficult. Diversity techniques such as frequency or time diversity, spatial diversity with the use of multiple antennas in multiple-input multiple output (MIMO) schemes, and cooperative diversity can be applied to improve the reliability of the signal in multipath channels. For instance, cooperative relaying, even with a single relay, can significantly improve the performance of the system; however, harsh environments would require the employment of numerous relays, and their placements could drastically affect the system performance, thus becoming a very challenging task [41]. Moreover, UAV relaying would present more complexities in the sense that the air-to-air link and the air-toground link are asymmetric and relaying happens in a 3D space. In [42], the use of multiple UAVs in relaying is tackled by studying the optimum positions of UAVs in relaying systems, equations for the best altitudes and distances are derived by maximizing the end-toend SNR. However, the coordination of multiple UAVs is also an intricate task. In [43], it was demonstrated that multiple antennas on the UAV provide a more robust radio channel in front of the antenna changes of orientation when the UAV is maneuvering. On the other hand, unlike narrowband channels, the UWB channel does not suffer from dense multipath propagation due to the inherent frequency diversity. However, a severe penalty in throughput and quality of service is experimented beyond short distances, then high datarate UWB communications systems are range restricted. Therefore, spatial diversity joint with UWB in UWBMIMO systems can lead to an increase signal-to-noise ratio (SNR), which can help to extend the range, reduce spatial power variations, and enable the use of high-level modulation for higher data rates [44]. Indeed, UWBMIMO systems have the potential to deliver extremely high data rates over short distances. However, even though multiple antennas on UAVs can bring improvements on coverage and diversity gains, it comes at the expense of increased computational capabilities, space, and power requirements.

3) Resource allocation and networking Maintaining connectivity of autonomous objects is crucial for suitable performance of the targeted tasks. Therefore, the communication between UAVs and between UAV and the infrastructure needs to be reliable in order to support the exchange of control messages, data offloading form sensors to UAVs, then among UAVs, and finally to the infrastructure [45]-[47]. Authors in [46] have evaluated a deployment composed of several UAVs, from which one is elected leader, and then aggregates the traffic from remaining UAVs and relays to the infrastructure or control center, while clusters members only connect to each other. Authors propose an resource subchannel allocation scheme which is shown to be NP-hard in urban environments. In a similar setting, [45] propose a 
solution in which spectrum sharing between UAV transmissions is allowed thus enhancing spectral efficiency (e.g. high data rates). In both works urban scenarios are assumed, hence we expect that under harsh conditions such allocation schemes might not be feasible, thus demanding dedicated resources form the network, or even dedicated architecture.

\section{Challenges in Sensor Fusion and Positioning}

As aforesaid, the proposed solution aims at autonomously flying UAVs, while still maintaining reliable wireless connection to edge servers to forward sensory data and offload computation. The target deployment scenario (caves) imposes difficult technological challenges due to its radio propagation features and harsh (inaccessible) environment for humans. In such situations, cyber physical systems which perform intended tasks autonomously based on current state and sensing with limited or even without human intervention are favored [48], [49]. To achieve that, the autonomous systems under investigation rely on information about network dynamics and environment features. Actually, it is necessary to simultaneously acquire, combine, and process large data base from various heterogeneous sources and then infer meaningful events and patterns to assist in the drone flight control and operation. It is also important to develop new positioning techniques for accurate 3D localization and reliable navigation of such unmanned vehicles.

Multidimensional sensor fusion combines sensory data from various heterogeneous sources so that the aggregated information offers more certainty than each component separately would [50]. This module has pivotal role for the overall solution as it acquires and processes information of many heterogeneous sources from non- and communication domains (e.g., laser technologies, video cameras, inertial measurement units and available transducers). For instance, UWB communication systems provide centimeter accuracy, strong multipath resistance and a good material penetration capability, which can be useful under Non-Line-of-Sight (NLoS) conditions due to its very large bandwidth. However, the transmission of information can be severely degraded under strong scattering conditions. Alternatively, radar systems implementing pulse compression techniques can significantly improve the range measurement precision if the effective bandwidth of the received signal is increased while keeping its energy constant [51], [52]. For combining such large and multidimensional data inside the sensor fusion component, the recursive Bayesian estimation approach (also known as Bayesian filtering) has been receiving a lot of attention lately [53], [54] as a viable solution to combine multidimensional data and enable positioning and tracking tasks, for example, Authors in [55]-[60] successfully applied these techniques in various deployment scenarios. A particularly useful approach is to combine Bayesian filtering with other machine learning models such as Gaussian processes [61], [62].

Equally important, modern radio access technologies such as 5G New Radio with large bandwidth, high carrier frequency, densely deployed base stations, and antenna arrays enable machine type communication in such a way that accurate positioning is provided by the radio network itself [63]-[65]. Hybrid strategies using heterogeneous sources of information and exploiting various metrics (such as time of flight, received signal strength, direction of arrival and combinations thereof) are of interest. As previously mentioned, the envisaged mechanisms rely on modern network architecture wherein communicating nodes offload not only sensory data, but also high demanding computational tasks to more capable edge servers that then estimate the target node position after gathering measurements and prior history [65], [66].

In the same way [67] addressed the prospects and key enabling technologies for highly efficient and accurate device positioning and tracking in $5 \mathrm{G}$ radio access networks. Building on the premises of ultra-dense networks as well as on the adoption of multicarrier waveforms and antenna arrays in the access nodes (ANs), the authors formulate extended Kalman filter (EKF)-based solutions for computationally efficient joint estimation and tracking of the time of arrival (ToA) and direction of arrival (DoA) of the user nodes (UNs) using uplink reference signals. Then, a second EKF stage was proposed in order to fuse the individual DoA and ToA estimates from one or several ANs into a UN position estimate. The computing complexity and energy consumption at the UN side were kept to a minimum. In results they demonstrated that by using their methods, sub-meter scale positioning and tracking accuracy of moving devices is indeed technically feasible in future $5 \mathrm{G}$. Positioning is a key enabler for new services in the upcoming wireless communication systems, for example, transportation, logistics, healthy care, animal husbandry, environmental monitoring and even wearables [58], [66], [68]. In the scenarios under consideration, access points, supporting localization units and low computation power terminals may also offload their computation to nearby edge server that actually estimate the target node position based on heterogeneous metrics and achieve better accuracy. Bayesian networks are employed here to capture the qualitative relationships between the random quantities aiming at obtaining valid posterior estimates for the unknown varieties of interest. The Markov Chain Monte Carlo (MCMC) method (a powerful generic computational approach) can be employed to approximately sample from arbitrary distributions [69]. Thus, we also resort to Bayesian probabilistic models to develop our collaborative localization procedure [70], [71]. In particular, the Hamiltonian Monte Carlo approach may be employed to carry out the MCMC method in a computationally efficient manner [54], [72].

\section{CONCLUDING REMARKS}

UAVs are becoming an attractive technology for various civil and military applications. However, indoor environments usually comprise passive objects that cause performance degradation, anomalous scattering, path loss, multipath fading, and interference effects. In this paper we present challenges and possible solutions for the use of UAVs in inhospitable environments by exemplifying the case of cave exploration. 
Although UAVs offer great opportunities for several sectors of our society through civil, commercial and military applications, their widespread adoption and seamless operation are still hindered by daunting technological challenges. As addressed in Section III-B, the target (indoor) deployment scenario imposes strong scattering conditions rendering an aggressive radio channel which limits the set of wireless radio access technologies capable of operating in such harsh circumstances. Therefore, further development is still needed to identify valid metrics (even non-communication based) to allow localization, mapping and navigation tasks. Typically, autonomous systems have limited computational and hardware capabilities, as well as limited power resources. Therefore, energy-efficient routing algorithms and network structure for sensory data an computation offloading in order to save battery time are needed, as well as effective charging techniques. In such applications, sensory data is crucial for proper autonomous system operation, so both real-time and stored data (from previous events and already discovered patterns) need to be properly combined to achieve accurate positioning and decision making, mainly in mission-critical applications. Moreover, the UAV popularization is still limited by the adoption of affordable materials and efficient technologies so as to reduce overall costs. Finally, UAV regulation by national aviation authorities may limit applications and deployment scenarios owing to mainly safety issues.

The addressed challenges provide unique guidelines that allow future researches in this field to continue looking for effective solutions, once UAVs are expected to be an important tool to improve the human quality of life.

\section{REFERENCES}

[1] S. G. Gupta, M. M. Ghonge, and P. M. Jawandhiya, "Review of Unmanned Aircraft System (UAS)," International Journal of Advanced Research in Computer Engineering \& Technology, vol. 2, no. 4, pp. 1646-1658, 2013, ISSN: 2278-1323.

[2] N. H. Motlagh, T. Taleb, and O. Arouk, "Low-Altitude Unmanned Aerial Vehicles-Based Internet of Things Services: Comprehensive Survey and Future Perspectives," IEEE Internet of Things Journal, vol. 3, no. 6, pp. 899-922, Dec 2016.

[3] E. Yagdereli, C. Gemci, and A. Z. Aktas, "A study on cyber-security of autonomous and unmanned vehicles," The Journal of Defense Modeling and Simulation: Applications, Methodology, Technology, vol. 12, no. 4, pp. 369-381, oct 2015. [Online]. Available: http://dx.doi.org/10.1177/1548512915575803

[4] G. H. Elkaim, F. A. P. Lie, and D. Gebre-Egziabher, Principles of Guidance, Navigation, and Control of UAVs. Dordrecht: Springer Netherlands, 2015, pp. 347-380.

[5] G. Balamurugan, J. Valarmathi, and V. P. S. Naidu, "Survey on UAV navigation in GPS denied environments," in International Conference on Signal Processing, Communication, Power and Embedded System (SCOPES). Paralakhemundi, India: IEEE, oct 2016, pp. 198-204.

[6] A. Morris, D. Ferguson, Z. Omohundro, D. Bradley, D. Silver, C. Baker, S. Thayer, C. Whittaker, and W. Whittaker, "Recent developments in subterranean robotics," Journal of Field Robotics, vol. 23, no. 1, pp. 35-57, 2006. [Online]. Available: https://doi.org/10.1002/rob.20106

[7] J. H. Kang, K. J. Park, and H. Kim, "Analysis of localization for dronefleet," in International Conference on Information and Communication Technology Convergence (ICTC). IEEE, Oct 2015, pp. 533-538.

[8] M. Cypriani, P. Canalda, and F. Spies, "Performance evaluation of a self calibrated 3D Wi-Fi fingerprinting positioning system," in Fourth International Conference on Communications and Electronics (ICCE). IEEE, Aug 2012, pp. 274-278.
[9] K. Li, C. Wang, S. Huang, G. Liang, X. Wu, and Y. Liao, "Selfpositioning for UAV indoor navigation based on 3D laser scanner, UWB and INS," in IEEE International Conference on Information and Automation (ICIA). IEEE, Aug 2016, pp. 498-503.

[10] F. J. Perez-Grau, F. Caballero, L. Merino, and A. Viguria, "Multi-modal mapping and localization of unmanned aerial robots based on ultrawideband and RGB-D sensing," in IEEE/RSJ International Conference on Intelligent Robots and Systems (IROS). IEEE, Sept 2017, pp. 34953502.

[11] "Human occupation of caves," in Encyclopedia of Caves and Karst Science, J. Gunn, Ed. Taylor \& Francis Books, 2003.

[12] "Surveying caves," in Encyclopedia of Caves and Karst Science, J. Gunn, Ed. Taylor \& Francis Books, 2003.

[13] P. Kambesis, "The importance of cave exploration to scientific research," Journal of cave and karst studies, vol. 69, no. 1, pp. 46-58, 2007.

[14] E. P. Canevese, P. Forti, A. Naseddu, L. Ottelli, and R. Tedeschi, "Laser Scanning Technology for the Hypogean Survey: the case of Santa Barbara Karst System (Sardinia, Italy)," Acta Carsologica, vol. 40, no. 1, pp. 65-77, 2011.

[15] M. Gallay, J. Kaňuk, Z. Hochmuth, J. D. Meneely, J. Hofierka, and V. Sedlák, "Large-scale and high-resolution 3-D cave mapping by terrestrial laser scanning: a case study of the Domica Cave, Slovakia," International Journal of Speleology, vol. 44, no. 3, pp. 277-291, 2015.

[16] R. Zlot and M. Bosse, "Three-dimensional mobile mapping of caves," Journal of Cave and Karst Studies, vol. 76, no. 3, p. 191, 2014.

[17] G. Zhang, B. Shang, Y. Chen, and H. Moyes, "SmartCaveDrone: 3D cave mapping using UAVs as robotic co-archaeologists," in International Conference on Unmanned Aircraft Systems (ICUAS). IEEE, June 2017, pp. 1052-1057.

[18] K. P. Valavanis, Advances in Unmanned Aerial Vehicles: State of the Art and the Road to Autonomy. Springer Publishing Company, Incorporated, 2007.

[19] H. Chao, Y. Cao, and Y. Chen, "Autopilots for small unmanned aerial vehicles: A survey," International Journal of Control, Automation and Systems, vol. 8, no. 1, pp. 36-44, Feb 2010.

[20] H. Wu, K. S. Tsakalis, and G. T. Heydt, "Evaluation of time delay effects to wide-area power system stabilizer design," IEEE Transactions on Power Systems, vol. 19, no. 4, pp. 1935-1941, 2004.

[21] B. Chaudhuri, R. Majumder, and B. C. Pal, "Wide-area measurementbased stabilizing control of power system considering signal transmission delay," IEEE Transactions on Power Systems, vol. 19, no. 4, pp. 1971-1979, 2004.

[22] V. Vittal, "Design of wide-area power system damping controllers resilient to communication failures," in 2016 IEEE Power and Energy Society General Meeting (PESGM). IEEE, 2016, pp. 1-1.

[23] T. Zhang, C.-X. Li, and Z.-L. Li, "Generalized predictive control and delay compensation for high-speed EMU network control system," in 2017 6th International Conference on Computer Science and Network Technology (ICCSNT). IEEE, 2017, pp. 511-515.

[24] K. Li and J. Baillieul, "Robust quantization for digital finite communication bandwidth (DFCB) control," IEEE Transactions on Automatic Control, vol. 49, no. 9, pp. 1573-1584, 2004.

[25] G. N. Nair and J. Baillieul, "Time to failure of quantized control via a binary symmetric channel," in Proceedings of the 45th IEEE Conference on Decision and Control. IEEE, 2006, pp. 2883-2888.

[26] A. A. Khuwaja, Y. Chen, N. Zhao, M. Alouini, and P. Dobbins, "A Survey of Channel Modeling for UAV Communications," IEEE Communications Surveys Tutorials, vol. 20, no. 4, pp. 2804-2821, 2018.

[27] D. W. Matolak and R. Sun, "Air-Ground Channel Characterization for Unmanned Aircraft Systems-Part I: Methods, Measurements, and Models for Over-Water Settings," IEEE Transactions on Vehicular Technology, vol. 66, no. 1, pp. 26-44, Jan 2017.

[28] R. Sun and D. W. Matolak, "Air-Ground Channel Characterization for Unmanned Aircraft Systems Part II: Hilly and Mountainous Settings," IEEE Transactions on Vehicular Technology, vol. 66, no. 3, pp. 19131925, March 2017.

[29] D. W. Matolak and R. Sun, "Air-Ground Channel Characterization for Unmanned Aircraft Systems-Part III: The Suburban and Near-Urban Environments," IEEE Transactions on Vehicular Technology, vol. 66, no. 8, pp. 6607-6618, Aug 2017.

[30] E. Yanmaz, S. Hayat, J. Scherer, and C. Bettstetter, "Experimental performance analysis of two-hop aerial 802.11 networks," in IEEE Wireless Communications and Networking Conference (WCNC), April 2014, pp. 3118-3123. 
[31] N. Ahmed, S. S. Kanhere, and S. Jha, "On the importance of link characterization for aerial wireless sensor networks," IEEE Communications Magazine, vol. 54, no. 5, pp. 52-57, May 2016.

[32] N. Goddemeier and C. Wietfeld, "Investigation of air-to-air channel characteristics and a uav specific extension to the rice model," in 2015 IEEE Globecom Workshops (GC Wkshps), Dec 2015, pp. 1-5.

[33] R. Amorim, H. Nguyen, P. Mogensen, I. Z. Kovács, J. Wigard, and T. B. Sørensen, "Radio channel modeling for uav communication over cellular networks," IEEE Wireless Communications Letters, vol. 6, no. 4, pp. 514-517, Aug 2017.

[34] A. Al-Hourani and K. Gomez, "Modeling cellular-to-uav path-loss for suburban environments," IEEE Wireless Communications Letters, vol. 7, no. 1, pp. 82-85, Feb 2018.

[35] A. E. Forooshani, S. Bashir, D. G. Michelson, and S. Noghanian, "A Survey of Wireless Communications and Propagation Modeling in Underground Mines," IEEE Communications Surveys Tutorials, vol. 15, no. 4, pp. 1524-1545, Fourth 2013.

[36] Z. Sun and I. F. Akyildiz, "Channel modeling and analysis for wireless networks in underground mines and road tunnels," IEEE Transactions on Communications, vol. 58, no. 6, pp. 1758-1768, June 2010.

[37] A. Hrovat, G. Kandus, and T. Javornik, "A survey of radio propagation modeling for tunnels," IEEE Communications Surveys Tutorials, vol. 16, no. 2, pp. 658-669, Second 2014.

[38] M. Boutin, A. Benzakour, C. L. Despins, and S. Affes, "Radio wave characterization and modeling in underground mine tunnels," IEEE Transactions on Antennas and Propagation, vol. 56, no. 2, pp. 540 549, Feb 2008.

[39] Q. P. Soo, S. Yong Lim, D. W. Gin Lim, K. Meng Yap, and S. Lun Lau, "Propagation measurement of a natural cave-turned-wine-cellar," IEEE Antennas and Wireless Propagation Letters, vol. 17, no. 5, pp. 743-746, May 2018.

[40] M. Hedley and I. Gipps, "Accurate wireless tracking for underground mining," in 2013 IEEE International Conference on Communications Workshops (ICC), June 2013, pp. 42-46.

[41] A. Crismani, S. Toumpis, U. Schilcher, G. Brandner, and C. Bettstetter, "Cooperative Relaying Under Spatially and Temporally Correlated Interference," IEEE Transactions on Vehicular Technology, vol. 64, no. 10, pp. 4655-4669, Oct 2015.

[42] Y. Chen, N. Zhao, Z. Ding, and M. Alouini, "Multiple UAVs as Relays: Multi-Hop Single Link Versus Multiple Dual-Hop Links," IEEE Transactions on Wireless Communications, vol. 17, no. 9, pp. 63486359, Sep. 2018.

[43] J. Romeu, A. Aguasca, J. Alonso, S. Blanch, and R. R. Martins, "Small UAV radiocommunication channel characterization," in Proceedings of the Fourth European Conference on Antennas and Propagation, April 2010, pp. 1-5.

[44] W. Q. Malik and D. J. Edwards, "Measured MIMO Capacity and Diversity Gain With Spatial and Polar Arrays in Ultrawideband Channels," IEEE Transactions on Communications, vol. 55, no. 12, pp. 2361-2370, Dec 2007.

[45] H. Hellaoui, O. Bekkouche, M. Bagaa, and T. Taleb, "Aerial Control System for Spectrum Efficiency in UAV-to-Cellular Communications," IEEE Communications Magazine, vol. 56, no. 10, pp. 108-113, oct 2018.

[46] S. Zhang, H. Zhang, B. Di, and L. Song, "Cellular UAV-to-X Communications: Design and Optimization for Multi-UAV Networks," IEEE Transactions on Wireless Communications, vol. 18, no. 2, pp. 13461359, feb 2019.

[47] B. Cao, L. Zhang, Y. Li, D. Feng, and W. Cao, "Intelligent Offloading in Multi-Access Edge Computing: A State-of-the-Art Review and Framework," IEEE Communications Magazine, vol. 57, no. 3, pp. 56-62, mar 2019.

[48] D. Floreano and R. J Wood, "Science, technology and the future of small autonomous drones," Nature, vol. 521, pp. 460-6, 052015.

[49] S. Thrun, S. Thayer, W. Whittaker, C. Baker, W. Burgard, D. Ferguson, D. Hahnel, D. Montemerlo, A. Morris, Z. Omohundro, C. Reverte, and Whittaker W, "Autonomous exploration and mapping of abandoned mines," IEEE Robotics Automation Magazine, vol. 11, no. 4, pp. 79-91, Dec 2004.

[50] B. Chandrasekaran, S. Gangadhar, and J. M. Conrad, "A survey of multisensor fusion techniques, architectures and methodologies," in SoutheastCon 2017, March 2017, pp. 1-8.

[51] J. A. Paredes, F. J. Álvarez, T. Aguilera, and J. M. Villadangos, “3D Indoor Positioning of UAVs with Spread Spectrum Ultrasound and
Time-of-Flight Cameras," Sensors, vol. 18, no. 1, p. 89, 2018. [Online]. Available: https://doi.org/10.3390/s18010089

[52] D. Sobers, G. Chowdhary, and E. Johnson, "Indoor Navigation for Unmanned Aerial Vehicles," in AIAA Guidance, Navigation, and Control Conference, Aug 2009.

[53] S. Särkkä, Bayesian filtering and smoothing. Cambridge University Press, 2013, vol. 3.

[54] A. Gelman, H. S. Stern, J. B. Carlin, D. B. Dunson, A. Vehtari, and D. B. Rubin, Bayesian data analysis. Chapman and Hall/CRC, 2013.

[55] Á. F. García-Fernández, L. Svensson, M. R. Morelande, and S. Särkkä, "Posterior linearization filter: Principles and implementation using sigma points," IEEE transactions on signal processing, vol. 63, no. 20, pp. 5561-5573, 2015

[56] Á. F. García-Fernández, L. Svensson, and S. Särkkä, "Cooperative localization using posterior linearization belief propagation," IEEE Transactions on Vehicular Technology, vol. 67, no. 1, pp. 832-836, 2018.

[57] A. F. Garcia-Fernandez, F. Tronarp, and S. Sarkka, "Gaussian target tracking with direction-of-arrival von Mises-Fisher measurements," IEEE Transactions on Signal Processing, 2019.

[58] D. Madigan, E. Einahrawy, R. P. Martin, W.-H. Ju, P. Krishnan, and A. Krishnakumar, "Bayesian indoor positioning systems," in Proceedings IEEE 24th Annual Joint Conference of the IEEE Computer and Communications Societies., vol. 2. IEEE, 2005, pp. 1217-1227.

[59] R. Kim, H. Lim, S.-N. Hwang, and B. O. Obele, "Robust indoor localization based on hybrid Bayesian graphical models," in 2014 IEEE Global Communications Conference. IEEE, 2014, pp. 423-429.

[60] K. F. Davies, I. G. Jones, and J. L. Shapiro, "A Bayesian Approach to Dealing with Device Heterogeneity in an Indoor Positioning System,' in 2018 International Conference on Indoor Positioning and Indoor Navigation (IPIN). IEEE, 2018, pp. 1-8.

[61] S. Sarkka, M. A. Alvarez, and N. D. Lawrence, "Gaussian process latent force models for learning and stochastic control of physical systems," IEEE Transactions on Automatic Control, 2018.

[62] S. Anderson, T. D. Barfoot, C. H. Tong, and S. Särkkä, "Batch nonlinear continuous-time trajectory estimation as exactly sparse gaussian process regression," Autonomous Robots, vol. 39, no. 3, pp. 221-238, Oct 2015. [Online]. Available: http://doi.org/10.1007/s10514-015-9455-y

[63] C. H. M. de Lima, J. Saloranta, and M. Latva-aho, "Collaborative Positioning Mechanism Using Bayesian Probabilistic Models for Industry Verticals," in ISWCS. IEEE, 2019.

[64] X. Lin, J. Bergman, F. Gunnarsson, O. Liberg, S. M. Razavi, H. S. Razaghi, H. Rydn, and Y. Sui, "Positioning for the internet of things: A 3gpp perspective," IEEE Communications Magazine, vol. 55, no. 12, pp. 179-185, Dec 2017.

[65] E. S. Lohan, M. Koivisto, O. Galinina, S. Andreev, A. Tolli, G. Destino, M. Costa, K. Leppanen, Y. Koucheryavy, and M. Valkama, "Benefits of Positioning-Aided Communication Technology in High-Frequency Industrial IoT," IEEE Communications Magazine, vol. 56, no. 12, pp. 142-148, December 2018.

[66] R. Di Taranto, S. Muppirisetty, R. Raulefs, D. Slock, T. Svensson, and H. Wymeersch, "Location-Aware Communications for 5G Networks: How location information can improve scalability, latency, and robustness of 5G," IEEE Signal Processing Magazine, vol. 31, no. 6, pp. 102-112, Nov 2014.

[67] M. Koivisto, M. Costa, J. Werner, K. Heiska, J. Talvitie, K. Leppänen, V. Koivunen, and M. Valkama, "Joint device positioning and clock synchronization in 5g ultra-dense networks," IEEE Transactions on Wireless Communications, vol. 16, no. 5, pp. 2866-2881, 2017.

[68] A. Nguyen, N. Milosavljevic, Q. Fang, J. Gao, and L. J. Guibas, "Landmark Selection and Greedy Landmark-Descent Routing for Sensor Networks," in 26th Annual Joint Conference of the IEEE Computer and Communications Societies (INFOCOM). IEEE, May 2007, pp. 661669.

[69] D. Gamerman and H. F. Lopes, Markov chain Monte Carlo: stochastic simulation for Bayesian inference. Chapman and Hall/CRC, 2006.

[70] D. Koller, N. Friedman, and F. Bach, Probabilistic graphical models: principles and techniques. MIT press, 2009.

[71] K. P. Murphy, "Machine Learning: A Probabilistic Perspective (Adaptive Computation and Machine Learning series)," 2018.

[72] S. Brooks, A. Gelman, G. Jones, and X.-L. Meng, Handbook of markov chain monte carlo. $\quad$ CRC press, 2011. 